\title{
Karnofksy Performance Status Not
} Evaluated

National Cancer Institute

\section{Source}

National Cancer Institute. Karnofksy Performance Status Not Evaluated. NCI Thesaurus.

Code C159863.

The Karnofsky Performance Status Score was not evaluated. 